\title{
Magnon-mediated Dzyaloshinskii-Moriya torque in homogeneous ferromagnets
}

\author{
Aurélien Manchon,,${ }^{1, *}$ Papa Birame Ndiaye, ${ }^{1}$ Jung-Hwan Moon, ${ }^{2}$ Hyun-Woo Lee,${ }^{3}$ and Kyung-Jin Lee ${ }^{2,4, \dagger}$ \\ ${ }^{1}$ King Abdullah University of Science and Technology (KAUST), Physical Science and Engineering Division, \\ Thuwal 23955-6900, Saudi Arabia \\ ${ }^{2}$ Department of Materials Science and Engineering, Korea University, Seoul 136-701, Korea \\ ${ }^{3}$ PCTP and Department of Physics, Pohang University of Science and Technology, Kyungbuk 790-784, Korea \\ ${ }^{4}$ KU-KIST Graduate School of Converging Science and Technology, Korea University, Seoul 136-713, Korea
}

(Received 5 January 2014; revised manuscript received 14 November 2014; published 1 December 2014)

\begin{abstract}
In thin magnetic layers with structural inversion asymmetry and spin-orbit coupling, the DzyaloshinskiiMoriya interaction arises at the interface. When a spin-wave current $\mathbf{j}_{m}$ flows in a system with a homogeneous magnetization $\mathbf{m}$, this interaction produces an effective fieldlike torque of the form $\mathbf{T}_{\mathrm{FL}} \propto \mathbf{m} \times\left(\mathbf{z} \times \mathbf{j}_{m}\right)$ as well as a dampinglike torque, $\mathbf{T}_{\mathrm{DL}} \propto \mathbf{m} \times\left[\left(\mathbf{z} \times \mathbf{j}_{m}\right) \times \mathbf{m}\right]$, the latter only in the presence of spin-wave relaxation ( $\mathbf{z}$ is normal to the interface). These torques mediated by the magnon flow can reorient the time-averaged magnetization direction and display a number of similarities with the torques arising from the electron flow in a magnetic two-dimensional electron gas with Rashba spin-orbit coupling. This magnon-mediated spin-orbit torque can be efficient in the case of magnons driven by a thermal gradient.
\end{abstract}

DOI: 10.1103/PhysRevB.90.224403

PACS number(s): 75.78.-n, 75.70.Tj, 75.75.-c, 75.76.+j

\section{INTRODUCTION}

Recent developments in condensed-matter physics have renewed the interest of the scientific community in the design and properties of materials with large spin-orbit coupling. Topics such as spin Hall effect [1], topological insulators [2], or skyrmions [3], all taking advantage of relativistic effects in solid state, have profoundly challenged our understanding of spin transport lately and present tremendously rich opportunities for innovative expansion of the research in condensedmatter systems. Utilizing spin-orbit coupling to enable the electrical manipulation of ferromagnets and magnetic textures has attracted a considerable amount of interest in the past few years [4-6]. The key mechanism, tagged spin-orbit torque, appears in ultrathin magnetic systems displaying inversion symmetry breaking such as (but not limited to) bilayers composed of heavy metals (such as Pt, Ta, W) and ferromagnets. The recent experimental results are interpreted in terms of Rashba [7] and spin Hall effect-induced torques [1] and the complexity of the spin transport in such systems is currently under intense investigations [8-10]. A major progress in this field has been to recognize the importance of Dzyaloshinskii-Moriya interaction (DMI) [11]. DMI results from spin-orbit coupling in structures with broken inversion symmetry and participates, in a number of systems, in the emergence of skyrmions and chiral spin textures [12-14]. Interestingly, DMI also arises from the interfacial spin-orbit coupling in ultrathin magnetic bilayers $[15,16]$ and results in chiral magnetic domain walls [14], providing an explanation to mysterious experimental behaviors such as current-induced domain-wall motion against the electron flow $[15,17,18]$.

In conjunction with electrically driven spin-orbit torques, another adjacent emerging research field aims at exploiting magnon flows and propagating spin waves instead of electrical carriers [19]. Indeed, magnons can carry spin currents [20],

\footnotetext{
*aurelien.manchon@kaust.edu.sa

${ }^{\dagger}$ kj_lee@korea.ac.kr
}

transmit information [21], and even control the motion of magnetic domain walls [22,23] and skyrmions [24]. The magnon flow may be driven by radio-frequency (rf) magnetic fields or temperature gradients [25], the latter being an important topic of the spin caloritronics field [26]. Recently, it has been realized that DMI impacts the propagation of spin waves just like spin-orbit coupling affects the electron flow, resulting in topological behaviors such as the magnon Hall effect and edge currents [27]. It was reported that the DMI effect on the spin-wave dispersion is similar to the Rashba spin-orbit coupling effect on electron dispersion [28-30]. Therefore, one anticipates that the spin-orbit torque due to electron flow in Rashba spin-orbit coupled systems might have its counterpart due to magnon flow in systems displaying DMI.

In this paper, we demonstrate that even in the absence of a magnetic texture, a magnon flow generates torques if magnons are subject to DMI just as an electron flow generates torques when submitted to Rashba interaction, even when the magnetization is homogeneous [8,9]. A direct consequence is the capability to tilt the magnetization direction of a homogeneous ferromagnet by applying a temperature gradient or a local rf field to generate the magnon flow (see Fig. 1). We show that merging the spin-orbit torques with spin caloritronics is rendered possible by the emergence of DMI in magnetic materials. The paper is organized as follows. The analytical derivation of the magnon-driven DzyaloshinskiiMoriya torque (DM torque) is developed in Sec. II. The central results of this work are presented in Sec. III, where the analytical expressions are compared with micromagnetic simulations. Section IV addresses the nature of this torque when driven by thermal gradients. Finally, the conclusion and perspectives are provided in Sec. V.

\section{ANALYTICAL DERIVATION OF THE DZYALOSHINSKII-MORIYA TORQUE}

Our objective is to analytically derive the static response of a magnetic system in the presence of both DMI and spin waves. To do so, we provide a general discussion on the response of a 


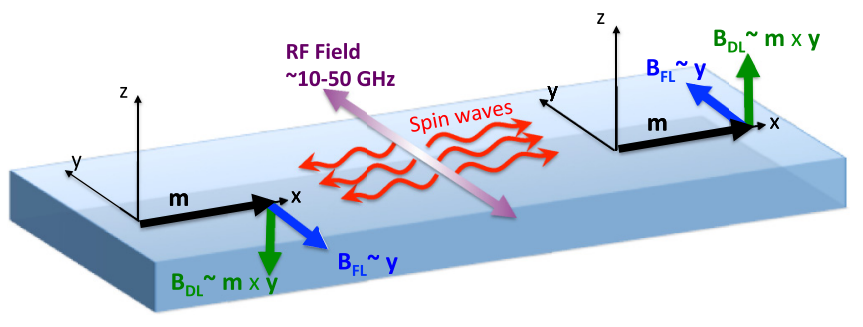

FIG. 1. (Color online) Schematics of the magnetized stripe studied in this work. The magnetization is initially oriented along $\mathbf{x}$ and spin waves are generated by an ac field applied in the center of the stripe at $x=0$. Due to DMI, the spin-wave flow induces effective fields, $\mathbf{B}_{\mathrm{FL}} \propto \mathbf{y}$ and $\mathbf{B}_{\mathrm{DL}} \propto \mathbf{m} \times \mathbf{y}$, resulting in deviations of the background magnetization $\Delta m_{y, z}$.

magnetic system submitted to a harmonic excitation, without assuming any specific form of the magnetic energy landscape, within the nonlinear spin-wave formalism (Sec. II A). This formalism is then applied to the specific case of DM torque (Sec. II B).

\section{A. Nonlinear spin-wave analysis}

\section{General considerations}

Let us first consider the case of a magnetization $\mathbf{m}$ subject to a harmonic excitation with frequency $\omega$ (such as spin waves or a time-dependent magnetic field). The magnetization $\mathbf{m}$ may be Fourier-expanded as follows:

$$
\begin{aligned}
\mathbf{m}= & \mathbf{m}_{(0)}+\left(\mathbf{m}_{(1)} e^{i \omega t}+\mathbf{m}_{(1)}^{*} e^{-i \omega t}\right)+\left(\mathbf{m}_{(2)} e^{2 i \omega t}+\mathbf{m}_{(2)}^{*} e^{-2 i \omega t}\right) \\
& +\mathcal{O}(\text { excitation amplitude })^{3},
\end{aligned}
$$

where $\mathbf{m}_{(0)}=\mathbf{m}_{(0)}(x) \equiv\langle\mathbf{m}\rangle$ denotes the time-independent magnetization profile and may slightly differ from the equilibrium profile due to the nonequilibrium correction arising from the nonlinear effect of the harmonic excitation. This excitationinduced correction is the central quantity that we aim to calculate. Here $\langle\ldots\rangle=\int_{T} \ldots d t / T$ denotes the temporal average over an oscillation period $T=2 \pi / \omega$.

The terms $\mathbf{m}_{(n)}=\mathbf{m}_{(n)}(x), n \geqslant 1$ are the Fourier components of the magnetization in time space, $\mathbf{m}_{(1)}$ representing the linear response of the system to the excitation, while $\mathbf{m}_{(n>1)}$ amounts to the nonlinear response of the system. Note that all the time-independent nonlinear responses are absorbed in $\mathbf{m}_{(0)}$. In the case of spin-wave excitations, $\mathbf{m}_{(1)}(x)$ describes the spatial profile of the spin-wave amplitude. Then, the linearization of the Landau-Lifshitz-Gilbert (LLG) equation fixes $\mathbf{m}_{(1)}$ up to its overall magnitude, which is nothing but the spin-wave amplitude (see below). On the other hand, $\mathbf{m}_{(2)}=\mathbf{m}_{(2)}(x)$ and the nonequilibrium correction part of $\mathbf{m}_{(0)}$ are of the order of (spin-wave amplitude) $)^{2}$ and describe the leading nonlinear corrections to the spin-wave profile when the spin-wave amplitude is not infinitesimally small. The $2 \omega$ and nonequilibrium 0 frequency components are of the order of (spin-wave amplitude) $^{2}$ because the squares of the first-order terms appear when the dynamics equations are expanded up to the second order. Since the first-order terms have frequency $\omega$, their squares generate $2 \omega$ and 0 frequency components, the latter being absorbed in the definition of $\mathbf{m}_{(0)}$. In Eq. (1), $\mathbf{m}_{(0)}$ is real whereas $\mathbf{m}_{(1)}$ and $\mathbf{m}_{(2)}$ are complex in general. Note that both sides of Eq. (1) are real.

The micromagnetic normalization condition

$$
\mathbf{m} \cdot \mathbf{m}=1
$$

imposes constraints on $\mathbf{m}_{(0)}, \mathbf{m}_{(1)}$, and $\mathbf{m}_{(2)}$. To extract these constraints, we expand Eq. (2) by using Eq. (1), up to the second order in spin-wave amplitude only. Analyzing the various Fourier components of the normalization condition gives the following set of equations:

$$
\begin{gathered}
\mathbf{m}_{(0)} \cdot \mathbf{m}_{(0)}=1-2 \mathbf{m}_{(1)}^{*} \cdot \mathbf{m}_{(1)}, \\
\mathbf{m}_{(1)} \cdot \mathbf{m}_{(0)}=\mathbf{m}_{(1)}^{*} \cdot \mathbf{m}_{(0)}=0, \\
2 \mathbf{m}_{(2)} \cdot \mathbf{m}_{(0)}=-\mathbf{m}_{(1)} \cdot \mathbf{m}_{(1)}, \\
2 \mathbf{m}_{(2)}^{*} \cdot \mathbf{m}_{(0)}=-\mathbf{m}_{(1)}^{*} \cdot \mathbf{m}_{(1)}^{*} .
\end{gathered}
$$

Equation (3) indicates that the magnitude of the timeindependent magnetization $\langle\mathbf{m}\rangle^{2}=\mathbf{m}_{(0)}^{2}$ is reduced below 1 due to the presence of spin waves (as expected from standard nonlinear spin-wave theory). Equation (4) states that $\mathbf{m}_{(1)}$ is orthogonal to $\mathbf{m}_{(0)}$, and Eqs. (5) and (6) show that the components of the second-order term $\mathbf{m}_{(2)}$ parallel to $\mathbf{m}_{(0)}$ are completely fixed by the normalization condition (2) once the first-order term $\mathbf{m}_{(1)}$ is fixed. But the components perpendicular to $\mathbf{m}_{(0)}$ are free from Eq. (2) and subject only to the LLG equation.

\section{Choice of an appropriate system of coordinates}

In order to model the impact of a flow of spin waves on the static direction of the magnetization $\mathbf{m}_{(0)}=\langle\mathbf{m}\rangle$, we need to adopt an appropriate system of coordinates that fulfills the constraints of micromagnetics. It is conventional to describe the magnetization direction using either the Cartesian [Fig. 2(a)] or the spherical coordinates system [Fig. 2(b)]. In the present work, we need to explicitly separate the time-independent part from the time-dependent part of the magnetization. In other words, we need to make an explicit (a)

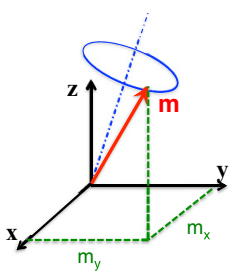

(b)

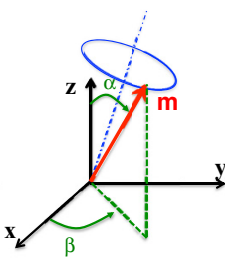

(c)

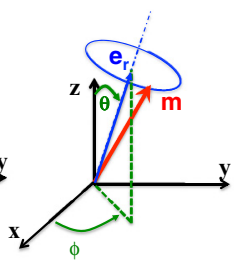

(d)
FIG. 2. (Color online) Different systems of coordinates, describing the general direction of a unitary vector $\mathbf{m}$. (a) In Cartesian coordinates, $\mathbf{m}=m_{x} \mathbf{e}_{x}+m_{y} \mathbf{e}_{y}+m_{z} \mathbf{e}_{z}$ and $m_{z}=\sqrt{1-m_{x}^{2}-m_{y}^{2}}$. (b) In spherical coordinates, $\mathbf{m}=\mathbf{e}_{r}$, where $\mathbf{e}_{r}=\cos \beta \sin \alpha \mathbf{e}_{x}+$ $\sin \beta \sin \alpha \mathbf{e}_{y}+\cos \alpha \mathbf{e}_{z}$. (c) In the system of coordinates we adopt in this work, the magnetization is defined as $\mathbf{m}=s_{r} \mathbf{e}_{r}+s_{\theta} \mathbf{e}_{\theta}+s_{\phi} \mathbf{e}_{\phi}$, where $s_{r}=\sqrt{1-s_{\theta}^{2}-s_{\phi}^{2}}$. Here, $\mathbf{e}_{r}$ is the direction of the axis about which the magnetization precesses. (d) Definition of the polar and azimuthal parts of the magnetization in (c), in a plane normal to the rotation axis $\mathbf{e}_{r}$. 
distinction between the static direction of precession and the time-dependent spin waves. To do so, we rewrite the magnetization as $\mathbf{m}=s_{r} \mathbf{e}_{r}+s_{\theta} \mathbf{e}_{\theta}+s_{\phi} \mathbf{e}_{\phi}$, where the timeindependent unit radial vector $\mathbf{e}_{r}$ is defined to be parallel to $\mathbf{m}_{(0)}$ and thus denotes the direction about which the magnetization precesses as sketched in Fig. 2(c). Then $\mathbf{m}_{(0)}=\left\langle s_{r}\right\rangle \mathbf{e}_{r}$. Once $\mathbf{e}_{r}$ is fixed, the associated polar unit vector $\mathbf{e}_{\theta}$ and azimuthal unit vector $\mathbf{e}_{\phi}$ are fixed. In spherical coordinates, they may be expressed as

$\mathbf{e}_{r}=\left(\begin{array}{c}\sin \theta \cos \phi \\ \sin \theta \sin \phi \\ \cos \theta\end{array}\right), \quad \mathbf{e}_{\theta}=\left(\begin{array}{c}\cos \theta \cos \phi \\ \cos \theta \sin \phi \\ -\sin \theta\end{array}\right), \quad \mathbf{e}_{\phi}=\mathbf{e}_{r} \times \mathbf{e}_{\theta}$.

Note that the angles $(\theta, \phi)$ as well as $\left(\mathbf{e}_{r}, \mathbf{e}_{\theta}, \mathbf{e}_{\phi}\right)$ are timeindependent by definition. By using the normalization conditions from the nonlinear spin-wave theory presented above, i.e., Eqs. (3)-(6), we deduce the following properties (up to the second order in spin-wave amplitude)

$$
\begin{aligned}
s_{r}\left\langle s_{r}\right\rangle= & \mathbf{m} \cdot \mathbf{m}_{(0)}=1-2 \mathbf{m}_{(1)}^{*} \cdot \mathbf{m}_{(1)} \\
& -\frac{1}{2}\left(\mathbf{m}_{(1)} \cdot \mathbf{m}_{(1)} e^{2 i \omega t}+\mathbf{m}_{(1)}^{*} \cdot \mathbf{m}_{(1)}^{*} e^{-2 i \omega t}\right), \\
s_{\theta}= & \mathbf{m} \cdot \mathbf{e}_{\theta}=\left(m_{(1), \theta} e^{i \omega t}+m_{(1), \theta}^{*} e^{-i \omega t}\right) \\
& +\left(m_{(2), \theta} e^{2 i \omega t}+m_{(2), \theta}^{*} e^{-2 i \omega t}\right), \\
s_{\phi}= & \mathbf{m} \cdot \mathbf{e}_{\phi}=\left(m_{(1), \phi} e^{i \omega t}+m_{(1), \phi}^{*} e^{-i \omega t}\right) \\
& +\left(m_{(2), \phi} e^{2 i \omega t}+m_{(2), \phi}^{*} e^{-2 i \omega t}\right),
\end{aligned}
$$

where we defined $\mathbf{m}_{(i)}=m_{(i), \theta} \mathbf{e}_{\theta}+m_{(i), \phi} \mathbf{e}_{\phi}$. From Eq. (7), one finds $\langle\mathbf{m}\rangle^{2}=\left\langle s_{r}\right\rangle^{2} \approx 1-2 \mathbf{m}_{(1)}^{*} \cdot \mathbf{m}_{(1)}$, which implies that the magnitude of the time-averaged magnetization is indeed smaller than 1. Equations (7)-(9) indicate that at the first order in spin-wave amplitude, $s_{r}$ is constant and $s_{\theta, \phi} \approx e^{ \pm i \omega t}$. We will see below that as long as $\mathbf{m}_{(0)}$ is determined up to the second order in the spin-wave amplitude, the $e^{ \pm 2 i \omega t}$ components of the excitations become irrelevant and thus may be ignored. Then the spin waves may be defined upon time averaging where the $e^{ \pm 2 i \omega t}$ components of the excitations disappear from the analysis. Therefore, in the spherical description adopted here, up to the first order in excitation amplitude, the spin waves are defined in the plane $\left(\mathbf{e}_{\theta}, \mathbf{e}_{\phi}\right)$, normal to the rotation direction $\mathbf{e}_{r}$ [see Fig. 2(d)].

Another important point concerns the degrees of freedom of our system. In Cartesian coordinates, Fig. 2(a), since $m_{x}^{2}+$ $m_{y}^{2}+m_{z}^{2}=1$ the system has only two degrees of freedom (say $m_{x}$ and $m_{y}$ ). In spherical coordinates, Fig. 2(b), the two degrees of freedom are the polar and azimuthal angles, $\alpha$ and $\beta$. In our system of coordinates, Fig. 2(c), the degrees of freedom are given by $s_{\theta}$ and $s_{\phi}$. Indeed, as will be explicitly shown below, the angles of the rotation axis, $\theta$ and $\phi$, are functions of the spin waves $s_{\theta}$ and $s_{\phi}$.

\section{From Landau-Lifshitz-Gilbert to Euler equations}

The LLG equation describing the dynamics of the system reads $\partial_{t} \mathbf{m}=\gamma \mathbf{m} \times \partial_{\mathbf{m}} W+\alpha \mathbf{m} \times \partial_{t} \mathbf{m}$, where $W$ is given in Eq. (17). Our objective is to determine the direction of the static magnetization under the influence of a flow of spin waves. To do so, we explicitly rewrite the LLG equation in terms of the spatial derivatives $\partial_{x} \theta, \partial_{x} \phi, \partial_{x}^{2} \theta, \partial_{x}^{2} \phi$. The two independent components (along $\mathbf{e}_{\theta}$ and $\mathbf{e}_{\phi}$ ) of the LLG equation adopt the general form

$$
\begin{aligned}
& \partial_{t} s_{\theta}+\alpha\left(s_{r} \partial_{t} s_{\phi}-s_{\phi} \partial_{t} s_{r}\right)=T_{\theta}^{(1)}+T_{\theta}^{(2)}+\cdots, \\
& \partial_{t} s_{\phi}-\alpha\left(s_{r} \partial_{t} s_{\theta}-s_{\theta} \partial_{t} s_{r}\right)=T_{\phi}^{(1)}+T_{\phi}^{(2)}+\cdots,
\end{aligned}
$$

where $T_{\theta, \phi}^{(i)}$ are the $i$ th order components (in spin-wave amplitude) of the term $\gamma \mathbf{m} \times \partial_{\mathbf{m}} W$ in the LLG equation. Since $\left\langle T_{\theta, \phi}^{(1)}\right\rangle=\left\langle\partial_{t} s_{\theta, \phi}\right\rangle=0$, it is clear that, up to the second order in spin-wave amplitude, the time averaging over one period of oscillation leads to

$$
\left\langle T_{\theta}^{(2)}\right\rangle=\alpha\left\langle s_{r} \partial_{t} s_{\phi}-s_{\phi} \partial_{t} s_{r}\right\rangle=\mathcal{O}(\text { excitation amplitude })^{4},
$$

$\left\langle T_{\phi}^{(2)}\right\rangle=-\alpha\left\langle s_{r} \partial_{t} s_{\theta}-s_{\theta} \partial_{t} s_{r}\right\rangle=\mathcal{O}(\text { excitation amplitude })^{4}$.

Indeed, since only time-dependent components of $s_{r}$ can couple with $s_{\theta}$ or $s_{\phi}$ to generate nonvanishing time-averaged values and since $s_{r}$ is time dependent only at the second order in excitation amplitude and above [see Eq. (7)], then the right-hand side of Eqs. (12) and (13) is nonzero only at the fourth order at least, and can therefore be neglected. The set of equations that determines the spatial profile of the static magnetization direction $(\theta, \phi)$ is

$$
\left\langle T_{\theta}^{(2)}\right\rangle=0, \quad\left\langle T_{\phi}^{(2)}\right\rangle=0 .
$$

Explicit expressions of Eq. (14) in terms of $\theta, \phi, s_{r}, s_{\theta}$, and $s_{\phi}$ are given in Sec. II B.

As will be explicitly shown below, Eq. (14) allows for the determination of $\theta$ and $\phi$ once $s_{r}, s_{\theta}$, and $s_{\phi}$ are determined only up to the linear order in spin-wave amplitude. This simplifies the calculation considerably. To the linear order in the spinwave amplitude, $s_{r} \approx 1$ and the linear equation of motion of the spin wave is then expressed in the form

$$
\begin{gathered}
\partial_{t} s_{\theta}+\alpha \partial_{t} s_{\phi}=T_{\theta}^{(1)}, \\
\partial_{t} s_{\phi}-\alpha \partial_{t} s_{\theta}=T_{\phi}^{(1)},
\end{gathered}
$$

that can be solved easily. The explicit expressions of Eqs. (14)-(16) being quite cumbersome in the general case, we only present below the solution when the magnetization initially lies along the longitudinal in-plane easy axis ( $x$ axis). The cases when the easy axis is in-plane transverse ( $y$ axis) or perpendicular to the plane ( $z$ axis) are treated in the Appendix.

\section{B. Magnetization deviations induced by the spin waves}

Let us consider a thin magnetic film with a magnetization $\mathbf{m}$ aligned along the in-plane easy axis ( $x$ axis) and subjected to an external ac magnetic field applied locally to make spin waves propagate along the $x$ axis, as displayed in Fig. 1. In this system, the magnetic energy reads

$$
\begin{aligned}
W= & A \sum_{i}\left(\partial_{i} \mathbf{m}\right)^{2}-D \mathbf{m} \cdot[(\mathbf{z} \times \nabla) \times \mathbf{m}] \\
& +2 \pi M_{s}^{2}(\mathbf{m} \cdot \mathbf{z})^{2}-K(\mathbf{m} \cdot \mathbf{x})^{2},
\end{aligned}
$$


where the first two terms are the symmetric exchange $(A)$ and antisymmetric Dzyaloshinskii-Moriya $(D)$ exchange energies, the last two terms are the demagnetizing $\left(2 \pi M_{s}^{2}\right)$ and the in-plane anisotropy $(K)$ energies, and $\nabla=\left(\partial_{x}, \partial_{y}, \partial_{z}\right)$. The form of the DMI we adopt here is derived for a cylindrically symmetric system with an interfacial inversion asymmetry along the normal $\mathbf{z}[13,15]$. All along the present study, we consider that the DMI is smaller than a certain threshold value $D_{c}\left[=\sqrt{2 A\left(K+2 \pi M_{s}^{2}\right)}\right]$ so that the uniformly magnetized state is energetically stable [29].

We assume that the magnetization is mostly oriented along the easy axis $\pm \mathbf{x}$. Then $\theta \rightarrow \pi / 2-\delta$ and $\phi \rightarrow \phi+(0, \pi)$ where $\delta, \phi \ll 1$, so that $\sin \theta \approx 1, \cos \theta \approx \delta$, and $\sin \phi \approx \eta \phi$, $\cos \phi \approx \eta$. Here, $\eta= \pm 1$ when the magnetization is along $\pm \mathbf{x}$. Since the linear effect of spin wave does not modify the equilibrium values of $\theta$ and $\phi, \delta$ and $\phi$ are of the second order in spin-wave amplitude at best. Up to the second order in spinwave amplitude, the time-averaged LLG equation reduces to $\gamma\left\langle\mathbf{m} \times \partial_{m} W\right\rangle=0$ [see Eq. (14)]. When expanded, the vector components of this equation become

$$
\begin{aligned}
& \left\langle s_{r}^{2}+s_{\phi}^{2}\right\rangle \partial_{x}^{2} \phi+\left\langle\partial_{x} s_{r}^{2}+\partial_{x} s_{\phi}^{2}\right\rangle \partial_{x} \phi-2\left\langle s_{\phi} \partial_{x} s_{\theta}\right\rangle \partial_{x} \delta \\
& =\frac{1}{\lambda^{2}}\left\langle s_{r}^{2}-s_{\phi}^{2}\right\rangle \phi+\eta \tilde{D}^{*}\left\langle s_{\phi} \partial_{x} s_{\theta}\right\rangle, \\
& \left\langle s_{r}^{2}+s_{\theta}^{2}\right\rangle \partial_{x}^{2} \delta+\left\langle\partial_{x} s_{r}^{2}+\partial_{x} s_{\theta}^{2}\right\rangle \partial_{x} \delta-2\left\langle s_{\theta} \partial_{x} s_{\phi}\right\rangle \partial_{x} \phi \\
& =\frac{1}{\lambda_{\mathrm{d}}^{2}}\left\langle s_{r}^{2}-s_{\theta}^{2}\right\rangle \delta-\eta \frac{\tilde{D}^{*}}{2}\left\langle\partial_{x} s_{r}^{2}+\partial_{x} s_{\theta}^{2}\right\rangle,
\end{aligned}
$$

where $\lambda^{2}=J / H_{k}, \lambda_{\mathrm{d}}^{2}=J /\left(H_{k}+H_{\mathrm{d}}\right)$ and $\tilde{D}^{*}=D^{*} / J$, with $J=2 A / M_{s}$. By considering $\left\langle s_{r}^{2}\right\rangle=1-\left\langle s_{\theta}^{2}+s_{\phi}^{2}\right\rangle$, Eqs. (18) and (19) become

$$
\begin{gathered}
\left(1-\left\langle s_{\theta}^{2}\right\rangle\right) \partial_{x}^{2} \phi-2\left(\left\langle s_{\theta} \partial_{x} s_{\theta}\right\rangle \partial_{x} \phi+\left\langle s_{\phi} \partial_{x} s_{\theta}\right\rangle \partial_{x} \delta\right) \\
=\frac{1}{\lambda^{2}}\left(1-\left\langle s_{\theta}^{2}\right\rangle-2\left\langle s_{\phi}^{2}\right\rangle\right) \phi+\eta \tilde{D}^{*}\left\langle s_{\phi} \partial_{x} s_{\theta}\right\rangle, \\
\left(1-\left\langle s_{\phi}^{2}\right\rangle\right) \partial_{x}^{2} \delta-2\left(\left\langle s_{\phi} \partial_{x} s_{\phi}\right\rangle \partial_{x} \delta+\left\langle s_{\theta} \partial_{x} s_{\phi}\right\rangle \partial_{x} \phi\right) \\
=\frac{1}{\lambda_{\mathrm{d}}^{2}}\left(1-2\left\langle s_{\theta}^{2}\right\rangle-\left\langle s_{\phi}^{2}\right\rangle\right) \delta+\eta \tilde{D}^{*}\left\langle s_{\phi} \partial_{x} s_{\phi}\right\rangle .
\end{gathered}
$$

These equations involve four types of terms: a second-order derivative of the angle (diffusionlike), a first-order derivative (wavelike), an anisotropy term, and a DMI term. We show below that this last term, proportional to the spin-wave flow $\left\langle s_{\phi} \partial_{x} s_{\phi, \theta}\right\rangle$, makes $\phi$ and $\delta$ nonvanishing, and drives the timeindependent components of the magnetization out of its rest position. In order to obtain a solvable equation, it is useful to count the order of each term in Eqs. (20) and (21). Considering that $s_{\theta}$ and $s_{\phi}$ are of the first order in the spin-wave amplitude, and $\phi$ and $\delta$ are of the second order in the spin-wave amplitude, one finds that only a few terms need to be retained for analysis up to the second order in the spin-wave amplitude.

For the sake of clarity, we now switch to the Cartesian coordinate system through the relation $\Delta m_{y} \equiv\left\langle s_{r}\right\rangle \sin \theta \sin \phi \approx$ $\eta \phi$ and $\Delta m_{z} \equiv\left\langle s_{r}\right\rangle \cos \theta \approx \delta$, where $\Delta m_{y, z}$ represent the time-averaged deviation of the magnetization induced by the flow of spin waves along the $\mathbf{y}$ and $\mathbf{z}$ axes. Those retained terms become

$$
\begin{gathered}
\partial_{x}^{2} \Delta m_{y}-\frac{1}{\lambda^{2}} \Delta m_{y}=\tilde{D}^{*}\left\langle s_{\phi} \partial_{x} s_{\theta}\right\rangle, \\
\partial_{x}^{2} \Delta m_{z}-\frac{1}{\lambda_{\mathrm{d}}^{2}} \Delta m_{z}=-\eta \tilde{D}^{*}\left\langle s_{\phi} \partial_{x} s_{\phi}\right\rangle,
\end{gathered}
$$

where we neglected higher orders in spin-wave amplitude $\left(s_{\theta, \phi}^{4} \ll s_{\theta, \phi}^{2} \ll 1\right)$ since the source term [right-hand side of Eqs. (22) and (23)] is already second order in $s_{\theta, \phi}$. In order to evaluate $\Delta m_{y}$ and $\Delta m_{z}$ from these equations, it is sufficient to determine $s_{\theta}$ and $s_{\phi}$ only up to the linear order in the spin-wave amplitude. For the latter, one just needs to deal with the linearized equations of motion of the spin wave [i.e., Eqs. (15) and (16)], which explicitly read

$$
\begin{gathered}
\partial_{t} s_{\theta}+\alpha \partial_{t} s_{\phi}=\gamma J \partial_{x}^{2} s_{\phi}-\gamma H_{k} s_{\phi}, \\
\partial_{t} s_{\phi}-\alpha \partial_{t} s_{\theta}=-\gamma J \partial_{x}^{2} s_{\theta}+\gamma\left(H_{k}+H_{d}\right) s_{\theta} .
\end{gathered}
$$

The solution is a spatially damped spin wave of the form

$$
\begin{gathered}
s_{\theta}=s_{\theta}^{0} e^{-|x| / 2 \Lambda} \cos (q x-\omega t), \\
s_{\phi}=s_{\phi}^{0} e^{-|x| / 2 \Lambda} \sin (q x-\omega t), \quad \Lambda=\gamma J q / \alpha \omega, \\
\omega=\gamma \sqrt{\left(J q^{2}+H_{d}+H_{k}\right)\left(J q^{2}+H_{k}\right)} .
\end{gathered}
$$

The absolute value $|x|$ ensures that the spin wave vanishes away from the source, taken at $x=0$. Now, by inserting Eqs. (26) and (27) into Eqs. (22) and (23), and taking the time average over a spin-wave precession period (i.e., $\left\langle s_{\phi} \partial_{x} s_{\phi}\right\rangle=$ $-\frac{\operatorname{sgn}(x)}{4 \Lambda}\left(s_{\phi}^{0}\right)^{2} e^{-|x| / \Lambda}$ and $\left.\left\langle s_{\phi} \partial_{x} s_{\theta}\right\rangle=-\frac{\operatorname{sgn}(x)}{2} q s_{\phi}^{0} s_{\theta}^{0} e^{-|x| / \Lambda}\right)$, we can track the impact of this damped spin wave on the deviations $\Delta m_{y, z}$. Considering that the right-hand sides of Eqs. (22) and (23) are odd functions of $x, \Delta m_{y, z}$ should vanish at $x=0$. Combined with the boundary condition, $\left.\Delta m_{y, z}\right|_{x \rightarrow \infty}=0$, one finds that

$$
\begin{gathered}
\Delta m_{y}=-\operatorname{sgn}(x) \frac{H_{\mathrm{DMF}}^{\mathrm{eff}}}{H_{\mathrm{k}}} \frac{\Lambda^{2}}{\Lambda^{2}-\lambda^{2}}\left(1-e^{-|x| / \lambda^{*}}\right), \\
\Delta m_{z}=\eta \operatorname{sgn}(x) \frac{H_{\mathrm{DMD}}^{\mathrm{eff}}}{H_{\mathrm{k}}+H_{\mathrm{d}}} \frac{\Lambda^{2}}{\Lambda^{2}-\lambda_{\mathrm{d}}^{2}}\left(1-e^{-|x| / \lambda_{\mathrm{d}}^{*}}\right),
\end{gathered}
$$

where $H_{\mathrm{DMF}}^{\mathrm{eff}}=D^{*} q s_{\theta}^{0} s_{\phi}^{0} e^{-|x| / \Lambda} / 2$ and $H_{\mathrm{DMD}}^{\mathrm{eff}}=D^{*}\left(s_{\phi}^{0}\right)^{2}$ $e^{-|x| / \Lambda} / 4 \Lambda$ and $\lambda_{(\mathrm{d})}^{*-1}=\lambda_{(\mathrm{d})}^{-1}-\Lambda^{-1}$. Note that $\Delta m_{y, z}$ are proportional to $D$ and (spin-wave amplitude) ${ }^{2}$, implying that these static magnetization deviations are consistent with the existence of effective magnetic fields along $\mathbf{y}$ and $\mathbf{z}$ directions, thus torques, generated by the magnon flow and the DMI. Considering that $\mathbf{m} \approx \eta \mathbf{x}$, the two field directions may be represented as $\mathbf{y}$ and $\mathbf{y} \times \mathbf{m}$, consistently with the absence (presence) of the factor $\eta$ in Eq. (28) [Eq. (29)]. The generated $D M$ fieldlike torque $(\mathrm{FLT}) \propto \mathbf{m} \times \mathbf{y}$ and $D M$ dampinglike torque $(\mathrm{DLT}) \propto \mathbf{m} \times(\mathbf{y} \times \mathbf{m})$ are in complete analogy with the Rashba torque $[8,9]$. Note also that $\Delta m_{y, z}$ are proportional to $\operatorname{sgn}(x)$, implying that in the two regions, $x>0$ and $x<0$, where the spin wave propagates in the opposite directions, the effective field signs are opposite. Thus the vectors $\mathbf{y}$ and $\mathbf{y} \times \mathbf{m}$ 
for the fields actually amount to $\mathbf{z} \times \mathbf{j}_{m}$ and $\left(\mathbf{z} \times \mathbf{j}_{m}\right) \times \mathbf{m}$, respectively, where $\mathbf{j}_{m}$ is the spin-wave current.

We emphasize that the torques derived above are obtained after time-averaging. Hence, the torques result in a static reorientation of the magnetization precession axis. Consequently, the present analysis does not preclude the emergence of any other DMI-induced mechanisms that could affect the overall magnetization dynamics (and therefore impact the motion of domain walls, for instance).

\section{MICROMAGNETIC SIMULATIONS}

To get further insights into the impact of propagating spin waves on the otherwise spatially homogeneous background magnetization, we now show micromagnetic simulation results for a semi-one-dimensional system (i.e., the system is discretized along the length direction with the unit cell size of $4 \mathrm{~nm}$ - total length of $16 \mu \mathrm{m}$ - but not along the width or the thickness direction). We solve the LLG equation with the magnetic energy functional given in Eq. (17). We define the gyromagnetic ratio $\gamma=1.76 \times 10^{7} \mathrm{Oe}^{-1} \mathrm{~s}^{-1}$, the saturation magnetization $M_{s}=800 \mathrm{emu} / \mathrm{cm}^{3}$, the exchange stiffness constant $A=1.3 \times 10^{-6} \mathrm{erg} / \mathrm{cm}$, and vary the easy axis anisotropy field $H_{\mathrm{k}}$, the demagnetization field along the thickness direction $H_{\mathrm{d}}$, and the damping constant $\alpha$. To excite spin waves, we apply an ac field $H_{\mathrm{ac}} \cos (2 \pi f t) \mathbf{y}$ to two unit cells at the center of the model system $(x=0)$ where $H_{\mathrm{ac}}=$ 100 Oe. This choice of the localized ac field is consistent with the situation assumed for Eqs. (28) and (29). We consider the absorbing boundary condition $[31,32]$ at the system edges to suppress spin-wave reflection.

Figure 3(a) shows the spatial distribution of the transverse projection of the magnetization direction $m_{y}$ for different DMI coefficients $D$. For $D=0$, the spatial distribution of $m_{y}$ is symmetric with respect to the spin-wave source $(x=0)$ and described by $m_{y} \cong 0+s_{y}(x) \cos (q|x|-\omega t)$, where " 0 " represents the $y$ component of the background magnetization and the spin-wave amplitude $s_{y}(x)$ decays with growing $|x|$ due to damping. For $D \neq 0$, on the other hand, the distribution acquires an antisymmetric component $\Delta m_{y}(x)$ with respect to $x=0$ and $m_{y} \cong \Delta m_{y}(x)+s_{y}(x) \cos (q|x|-\omega t)$, according to Fig. 3(a). Thus the propagating spin wave modifies the $y$ component of the background magnetization from 0 to a nonzero time-averaged value $\Delta m_{y}(x)$, which is shown in Fig. 3(b) for a background magnetization initially lying along $+\mathbf{x}$. Reversing the direction of the background magnetization along $-\mathbf{x}$ does not change the sign of $\Delta m_{y}$ (not shown). Thus the simulation result for $\Delta m_{y}$ is qualitatively consistent with the vector expression $-\mathbf{z} \times \mathbf{j}_{m}$ obtained from Eq. (28). For a quantitative comparison, Fig. 3(b) shows the deviation $\Delta m_{y}$ for various values of $\alpha$, where symbols are numerical results and lines are obtained from Eq. (28). When $s_{\theta}^{0}$ and $s_{\phi}^{0}$ in $H_{\mathrm{DMF}}^{\text {eff }}$ are determined from the numerical simulation result at $x=0$, the analytical expression reproduces the numerical results very well for the entire range of $x$.

Another intriguing observation is the emergence of the DMDLT $[\propto \mathbf{m} \times(\mathbf{y} \times \mathbf{m})]$ that induces an out-of-plane deviation $\Delta m_{z}$. Figure 3(c) shows the spatial distribution of $\Delta m_{z}$ for
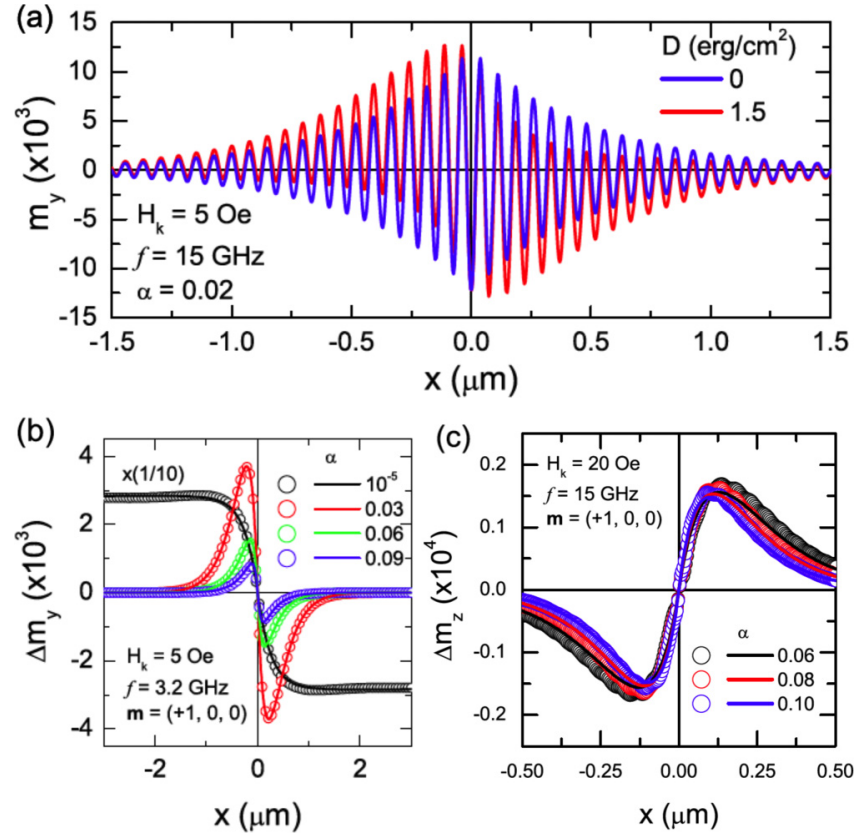

FIG. 3. (Color online) Numerical results for magnon-mediated Dzyaloshinskii-Moriya fieldlike and dampinglike torques. (a) Spatial distribution of the normalized $y$ component of magnetization $\left(=m_{y}\right)$ for $D=0$ (blue) and $D=1.5 \mathrm{erg} / \mathrm{cm}^{2}$ (red). (b) Normalized magnetization tilting $\Delta m_{y}$ for various damping constants $\alpha$ when the magnetization initially lies along $+\mathbf{x}$ for $D=1.5 \mathrm{erg} / \mathrm{cm}^{2}$ calculated numerically (open symbols) and using Eq. (8) (solid lines). The results with $\alpha=10^{-5}$ are multiplied by $1 / 10$. (c) Normalized magnetization tilting $\Delta m_{z}$ for various damping constants $\alpha$ when the magnetization initially lies along $+\mathbf{x}$, for $D=1.5 \mathrm{erg} / \mathrm{cm}^{2}$ calculated numerically (open symbols) and using Eq. (9) (solid lines). Here we assume $H_{d}=0$. When the magnetization is switched to the $-\mathbf{x}$ direction, panel (b) remains unchanged while the curves in panel (c) are mirrored with respect to the $\mathbf{x}$ axis.

different $\alpha$ when the magnetization is initially aligned along $+\mathbf{x}$. For clarity, we assume $H_{\mathrm{d}}=0$ to make $\lambda_{\mathrm{d}}$ and $\Delta m_{z}$ larger. The numerical results (open symbols) are in good agreement with Eq. (29) (solid lines) and $\Delta m_{z}$ consistently changes sign with the magnetization direction (not shown). As demonstrated by Fig. 3(c) and Eq. (29), the DM-DLT is proportional to the damping constant $\alpha$ (since $\Lambda \propto 1 / \alpha$ ), which echoes the nonadiabatic correction to the electronic-spin torque in the presence of spin-flip relaxation, as proposed by Zhang et al.in magnetic textures and spin valves [33]. In metallic systems, the spin relaxation modifies the spin dynamics of the itinerant electrons which results in an additional torque component of the form $-\beta \mathbf{m} \times \boldsymbol{\tau}$, where $\boldsymbol{\tau}$ is the torque in the absence of spin relaxation and $\beta$ is proportional to the spin-relaxation rate [33]. The same effect is at the origin of the nonadiabatic torque in electron-driven and magnondriven magnetic excitations: the magnetic damping $\alpha$ not only attenuates the spin-wave current, but also relaxes the spin polarization carried by the spin waves producing the additional dampinglike torque proportional to $H_{\mathrm{DMD}}^{\text {eff }}$. As a result, the overall magnitude of $\Delta m_{z}$ vanishes in the limit of zero damping. 

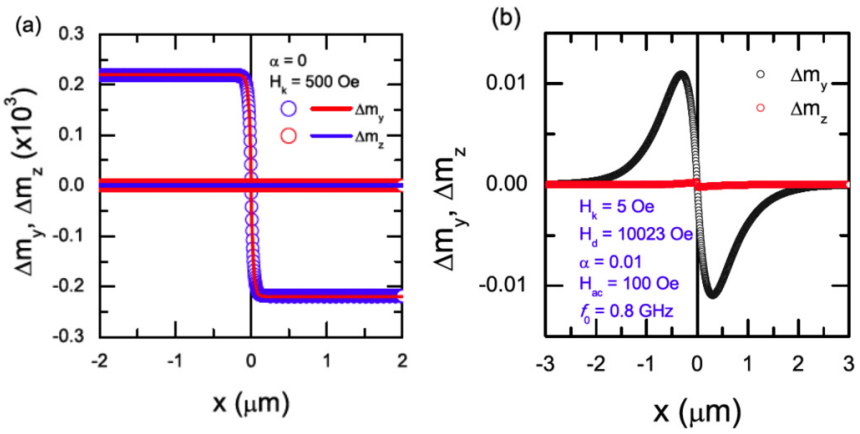

FIG. 4. (Color online) (a) Numerical results of spin-waveinduced magnetization tilting $\Delta m_{y}$ and $\Delta m_{z}$ with $\alpha=0$. Symbols and lines are obtained from numerical simulation and Eqs. (30) and (31), respectively. (b) $\Delta m_{y}$ and $\Delta m_{z}$ obtained with $\alpha=0.01$, $H_{k}=5 \mathrm{Oe}, H_{a c}=100 \mathrm{Oe}, H_{d}=10023 \mathrm{Oe}, D=1.5 \mathrm{erg} / \mathrm{cm}^{2}$, and $f=0.8 \mathrm{GHz}$. The magnitude of the deviation is about $1 \%$.

In order to further demonstrate the distinctive role of spinwave flow and spin-wave relaxation on the two components of the DM torque, we consider the reaction of an undamped magnetic system to the spin-wave flow in the vanishing small Gilbert damping coefficient, $\alpha \rightarrow 0$. In this limit, the previous expressions for $\Delta m_{y, z}$ reduce to

$$
\begin{gathered}
\Delta m_{y}=-\operatorname{sgn}(x) \frac{D^{*} q s_{\theta}^{0} s_{\phi}^{0}}{2 H_{\mathrm{k}}}\left(1-e^{-|x| / \lambda}\right), \\
\Delta m_{z}=0 .
\end{gathered}
$$

We performed numerical simulations with $\alpha=0, H_{k}=$ $500 \mathrm{Oe}, H_{a c}=100 \mathrm{Oe}, H_{d}=10023 \mathrm{Oe}, D=1.5 \mathrm{erg} / \mathrm{cm}^{2}$, and $f=15.9 \mathrm{GHz}$ [see Fig. 4(a)]. Simulations with $\alpha=10^{-5}$ were performed to check that the zero-damping calculation is free from unstable numerical errors (not shown). In both cases, we find that $\Delta m_{y}$ becomes constant when $|x| \gg \lambda(\approx 0.1 \mu \mathrm{m}$ here), whereas $\Delta m_{z}$ vanishes completely. The numerical results are in agreement with Eqs. (30) and (31), which proves that the magnetization tilting $\Delta m_{y}$ is caused not by the spin relaxation, but by the spin-wave flow itself. In contrast, $\Delta m_{z}$ vanishes in the vanishing damping limit, proving that this deviation is induced by the spin-wave relaxation.

Finally, to test the feasibility of detecting the tilting experimentally, we performed numerical simulation with $\alpha=$ $0.01, H_{k}=5$ Oe, $H_{a c}=100$ Oe, $H_{d}=10023$ Oe, $D=$ $1.5 \mathrm{erg} / \mathrm{cm}^{2}$, and $f=0.8 \mathrm{GHz}$ [see Fig. 4(b)]. We note that the vertical axis is not scaled so that $\Delta m_{y}$ reaches about $1 \%$ of the saturation magnetization, which corresponds to a DM field of 0.15 Oe. This tilt can be detected by either an electrical or optical (Kerr effect) way, after averaging the magnetization direction over time. For instance, using MOKE microscopy Fan et al. [34] measured a laser polarization change of about 1 microrad, corresponding to a magnetization tilting of $0.1 \%$. In Fig. 1 of Ref. [34], the effective field corresponding to the dampinglike torque is about $1.1 \mathrm{Oe}$. We also note that Ref. [35] shows a MOKE sensitivity of 60 nanorad (see Fig. 2 of Ref. [35]). These works indicate that the deviations predicted in the present work should be detectable experimentally.

\section{THERMALLY INDUCED DZYALOSHINSKII-MORIYA TORQUE}

\section{A. Analogy with a two-dimensional Rashba gas}

So far we have discussed the effect of the spin wave $\psi_{m}=s_{\theta}+i s_{\phi}$ on $\Delta m_{y, z}$. Still further insight can be gained by considering the effect of $\Delta m_{y, z}$ on $\psi_{m}$. For this, we go beyond the linearized LLG equation and introduce to Eqs. (24) and (25) the lowest-order coupling terms between $\Delta m_{y, z}$ and $\psi_{m}$, which are linear to both $\Delta m_{y, z}$ and $\psi_{m}$. In the short-wavelength regime, where the exchange and DM interactions dominate over the anisotropy, the resulting equations fall into the following form of the effective Schrödinger equation:

$$
i \hbar \partial_{t} \psi_{m}=\hat{H}_{m} \psi_{m}=\left(\frac{\hat{\mathbf{p}}^{2}}{2 m^{*}}+\frac{\alpha_{\mathrm{DM}}}{\hbar} \hat{\mathbf{p}} \cdot(\mathbf{z} \times \mathbf{m})\right) \psi_{m},
$$

where $\hat{\mathbf{p}}=-i \hbar \nabla$ is the momentum operator, $m^{*}=$ $\hbar M_{s} / 4 \gamma A$ is the magnon mass, and $\alpha_{\mathrm{DM}} / \hbar=2 \gamma D / M_{s}$ is Dzyaloshinskii-Moriya velocity for the spin waves. This equation instructively resembles Schrödinger's equation of an itinerant electron spin in a homogeneous magnetic twodimensional electron gas in the presence of Rashba spin-orbit coupling [7],

$$
i \hbar \partial_{t} \psi_{e}=\left(\frac{\hat{\mathbf{p}}^{2}}{2 m}+\frac{\alpha_{\mathrm{R}}}{\hbar} \hat{\mathbf{p}} \cdot(\mathbf{z} \times \hat{\boldsymbol{\sigma}})+J_{\mathrm{ex}} \mathbf{m} \cdot \hat{\boldsymbol{\sigma}}\right) \psi_{e},
$$

where $\alpha_{\mathrm{R}}$ is the Rashba spin-orbit coupling and $J_{\text {ex }}$ is the $s-d$ exchange between itinerant electron spins $\hat{\sigma}$ and the local moments aligned along $\mathbf{m}$. Equations (32) and (33) differ by the presence of the $s-d$ exchange term. Indeed, in contrast with electron spins, the magnon spin is by definition aligned along the local magnetization direction and its wave function $\psi_{m}$ is not a two-component spinor. Nevertheless, their similarity implies that properties of the Rashba system, such as current-induced Rashba field (also called inverse spin galvanic effect) of the form [8] $\mathbf{H}_{\mathrm{R}}=-\alpha_{\mathrm{R}} m \mathbf{z} \times \mathbf{j}_{s} / \hbar M_{s}\left(\mathbf{j}_{s}\right.$ being the flowing spin current), are at least partly enabled by the presence of DMI in magnonic systems. The propagating spin wave and background magnetization $\mathbf{m}$ interact through the energy term $\left(\alpha_{\mathrm{DM}} / \hbar\right)\langle\hat{\mathbf{p}}\rangle_{m} \cdot(\mathbf{z} \times \mathbf{m})$, where $\langle\ldots\rangle_{m}$ denotes the quantum average on the magnon state $\psi_{m}$. This interaction term yields a torque of the form

$$
\mathbf{T}_{\mathrm{FL}}=\gamma \mathbf{m} \times \partial_{\mathbf{m}}\left\langle\hat{H}_{m}\right\rangle=\mathbf{m} \times\left\{\frac{\alpha_{\mathrm{DM}}}{\hbar} \mathbf{z} \times\langle\mathbf{p}\rangle_{m}\right\} .
$$

The expression within the brackets $\{\ldots\}$ is nothing but the effective field of the DM-FLT, $\mathbf{H}_{\mathrm{DMF}}$, and $\langle\mathbf{p}\rangle_{m}$ amounts to the spin-wave current. The DM-DLT can be obtained qualitatively by considering the correction due to the damping on the spinwave dynamics [see Eqs. (24) and (25)]. In a Landau-Lifshitz approach, the magnetic damping corrects the torque by adding a contribution of the form $\gamma \alpha \mathbf{m} \times\left(\mathbf{m} \times \mathbf{H}_{\mathrm{DMF}}\right)$ that produces the DM-DLT term.

\section{B. Thermally driven Dzyaloshinskii-Moriya torque}

We next discuss the DM torque arising from the flow of magnons generated by a thermal gradient. The spin-wave flow generated by rf field has two major drawbacks: (i) the wavelength $2 \pi / q$ of the spin wave is rather large ( $74 \mathrm{~nm}$ in the 
present study) producing a very small effective field $\left[H_{\mathrm{DMF}}^{\text {eff }}<\right.$ 0.1 Oe in Fig. 4(b)] and (ii) the magnon flow (hence, the DM torque) vanishes away from the rf source over the attenuation length $\Lambda$. Therefore, thermal magnons driven by a uniform temperature gradient, $\nabla T$, are interesting candidates for the proposed effect. Indeed, they possess a shorter wavelength and can be maintained over the region where the thermal gradient is applied. Following Eq. (34), these magnons exert a torque of the form $\mathbf{T}_{\mathrm{FL}}^{\mathrm{th}}=\left(\alpha_{\mathrm{DM}} m^{*} / \hbar M_{s}\right) \mathbf{m} \times\left(\mathbf{z} \times \mathbf{j}_{m}\right)$ on the magnetization, where $\mathbf{j}_{m}$ is the magnon flow and can be estimated using a phenomenological Boltzmann equation [36],

$$
\mathbf{j}_{m}=-\frac{k_{B} \partial_{x} T}{2 \pi \alpha}\left[\int_{K V / k_{B} T}^{T_{c} / T} \frac{x e^{x} d x}{\left(e^{x}-1\right)^{2}}\right] \mathbf{x} .
$$

To evaluate the amount of magnonic current flowing through the system, we choose reasonable parameters for YIG as found in the literature [22,37]: $\alpha=10^{-4}, V \approx 1.9 \mathrm{~nm}^{3}$, $K \approx 2 \times 10^{5} \mathrm{erg} / \mathrm{cm}^{3}, T_{c}=550 \mathrm{~K}, T=300 \mathrm{~K}$, and $\partial_{x} T=$ $20 \mathrm{~K} / \mathrm{mm}$. Under these conditions, for a 1-nm-thick magnetic slab, the flow of magnons is $j_{m} \approx 2.29 \times 10^{24} \mathrm{~s}^{-1} \mathrm{~cm}^{-1}$, two orders of magnitude smaller than the usual critical switching particle current in conventional spin-transfer torque configuration $\left(\approx 10^{26} \mathrm{~s}^{-1} \mathrm{~cm}^{-2}\right)$. The effective magnetic field generated by this magnon flow is $H_{\mathrm{FL}}=\hbar D /\left(2 A M_{s}\right) j_{x} \approx$ $1.7 \mathrm{Oe}$, which is at least one order of magnitude larger than the effective field obtained from rf spin waves in this work. This conservative estimation leaves plenty of room for improvement such as (i) increasing the temperature gradient, (ii) reducing the magnetization damping, (iii) decreasing the magnetic anisotropy, and (iv) increasing the DMI.

\section{Simulations with a temperature gradient}

We performed numerical simulations with a uniform temperature gradient by including the thermal fluctuation fields, i.e., the Gaussian-distributed random fluctuation fields $\mathbf{h}(t)$
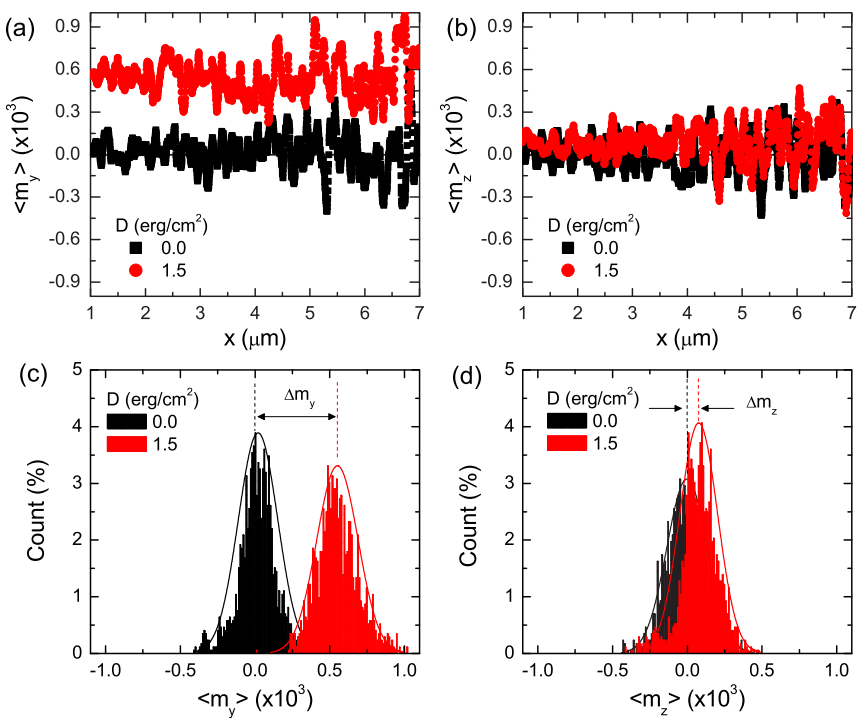

FIG. 5. (Color online) Magnetization tilting induced by uniform temperature gradient. Spatial distribution of time-averaged (a) $\left\langle m_{y}\right\rangle$ and (b) $\left\langle m_{z}\right\rangle$. Corresponding histograms of (c) $\left\langle m_{y}\right\rangle$ and (d) $\left\langle m_{z}\right\rangle$. $\left(\langle\mathbf{h}(t)\rangle=0,\left\langle h_{i}(t) h_{j}(t+\delta t)\right\rangle=2 \alpha k_{B} T /\left(\gamma M_{S} V \delta t\right) \delta_{i j}\right.$, where $i, j$ are the Cartesian coordinates, $V$ is the unit-cell volume, and $\delta t$ is the integration time step [38]). We assume $\alpha=0.1$, $H_{k}=500 \mathrm{Oe}, H_{d}=0 \mathrm{Oe}$, the nanowire width of $100 \mathrm{~nm}$, and $\partial_{x} T=0.025 \mathrm{~K} / \mathrm{nm}$. $T$ is set to be zero at $x=0$ and increases with $x$. For simplicity, we ignored the temperature dependence of other magnetic parameters and also their temperaturedependent renormalization [39]. As a result, the magnitude of the effect reported below should be regarded only as an order of magnitude estimation. Figures 5(a) and 5(b) show the time-averaged $m_{y}$ and $m_{z}$ components with varying $D$. The averaged magnetization components fluctuate around " 0 " for $D=0$, whereas they fluctuate around clearly nonzero value for nonzero $D$. Figures 5(c) and 5(d) are histograms corresponding to Figs. 5(a) and 5(b), respectively, which show the finite magnetization tiltings induced by the temperature gradient. The effective field generated by the thermally induced DM torque is $H_{\mathrm{DMF}}^{t h} \equiv H_{k} \Delta m_{y} \approx 0.25$ Oe.

\section{DISCUSSION AND CONCLUSION}

In this paper, we demonstrated that Dzyaloshinskii-Moriya interaction mediated by spin waves can generate a torque on a homogeneous magnetization that resembles the Rashba torque, its electronic counterpart, displaying both fieldlike and dampinglike components. The torque is expected to be much more efficient in the case of a magnon flow driven by a thermal gradient than for a standard rf-excited spin wave. It is important to stress that our results are not limited to systems displaying interfacial DMI but can be also extended to materials accommodating bulk DMI since the energy functional needs only to display an antisymmetric exchange term $\sum_{i j} \mathcal{D}_{i j} \cdot \mathbf{S}_{i} \times \mathbf{S}_{j}$, such as in pyrochlore crystals [27], chiral magnets [40], and multiferroics [41].

The recent realization of a magnonic transistor based on a magnetic insulator has confirmed the ability of spin waves for low energy consuming logic operations [42]. Since the information process does not require charge currents nor electric voltages, it is expected to reduce the energy losses due to Joule heating. The present work builds up a bridge between spin-orbit transport, magnonics, and spin caloritronics and extends the properties of spin waves to systems without inversion symmetry. This mechanism enables the magnonic control of the magnetization direction in ferromagnets without the use of charge currents or electrical voltages and is expected to be detectable in systems ranging from thin magnetic bilayers to bulk noncentrosymmetric crystals. It therefore opens promising avenues in the development of chargeless information technology.

\section{ACKNOWLEDGMENTS}

The authors acknowledge fruitful discussions with M. D. Stiles and K. W. Kim. A.M. and P.B.N. were supported by the King Abdullah University of Science and Technology (KAUST). K.J.L. was supported by NRF (Grant No. 2013R1A2A2A01013188). H.W.L. was supported by NRF (Grants No. NRF-2013R1A2A2A05006237 and No. 20110030046). 


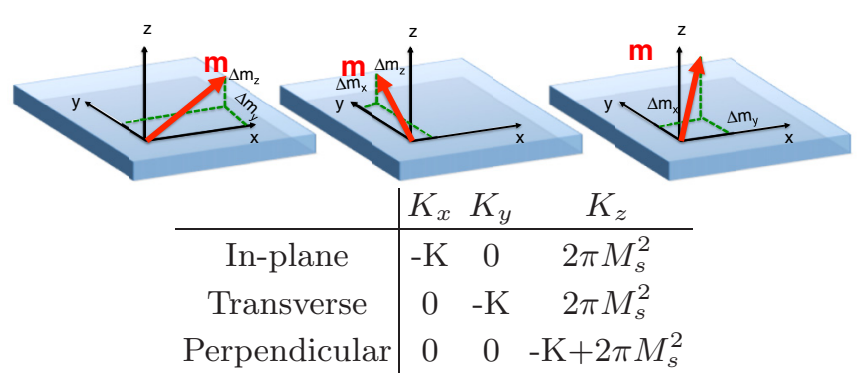

FIG. 6. (Color online) (Top panel) Three magnetic configurations investigated in this work and their corresponding anisotropy energy constants. At rest (i.e., in the absence ofmagnon flow), the magnetization is assumed to be aligned either along the easy axis, which is $x$ (in-plane longitudinal anisotropy), $y$ (in-plane transverse anisotropy), or $z$ (perpendicular anisotropy). (Bottom panel) The table provides the values of anisotropy constants for the three cases depicted above.

\section{APPENDIX}

In this Appendix, we analytically derive the DM torque in the case of in-plane transverse anisotropy (i.e., along the $y$ direction) and perpendicular anisotropy (i.e., along the $z$ direction). Let us start by generalizing our approach and consider a one-dimensional magnetic system described by the following energy functional:

$W=A \sum_{i}\left(\partial_{i} \mathbf{m}\right)^{2}-D \mathbf{m} \cdot[(\mathbf{z} \times \nabla) \times \mathbf{m}]+\sum_{i} K_{i}\left(\mathbf{m} \cdot \mathbf{e}_{i}\right)^{2}$,

where the first term is the symmetric exchange energy $(A)$ and the second term is the antisymmetric DzyaloshinskiiMoriya exchange energy $(D)$. The last term $\left(K_{i}\right)$ is a general anisotropy term that accounts for the magnetic anisotropy and demagnetizing energy along the three Cartesian directions $\mathbf{e}_{x, y, z}$. More explicitly, Fig. 6 provides the actual values of $K_{i}$ in the case of in-plane longitudinal anisotropy, in-plane transverse anisotropy, and perpendicular anisotropy.

The case of in-plane longitudinal anisotropy is treated in the main text. In this case, $K_{x}=-K, K_{y}=0$, and $K_{z}=$ $2 \pi M_{s}^{2}=K_{d}$. We here treat the two remaining cases of in-plane transverse anisotropy and perpendicular anisotropy.

\section{In-plane transverse anisotropy}

In this case, $K_{x}=0, K_{y}=-K$, and $K_{z}=2 \pi M_{s}^{2}=K_{d}$. We assume that the magnetization is mostly oriented along the easy axis $\pm \mathbf{y},(\theta, \phi) \rightarrow(\pi / 2-\delta, \eta \pi / 2-\varphi)$ where $\delta, \varphi \ll 1$, so that $\sin \theta \approx 1, \cos \theta \approx \delta$ and $\sin \phi \approx \eta, \cos \phi \approx \eta \varphi(\eta= \pm 1)$. Again $\delta$ and $\varphi$ are of the second order in spin-wave amplitude. Following the same procedure as above, and defining $\Delta m_{x}=$ $\eta \varphi, \Delta m_{z}=\delta$, we obtain up to the second order in spin-wave amplitude

$$
\begin{aligned}
& \partial_{x}^{2} \Delta m_{x}-\tilde{D}^{*} \partial_{x} \Delta m_{z}-\frac{1}{\lambda^{2}} \Delta m_{x}=0, \\
& \partial_{x}^{2} \Delta m_{z}+\tilde{D}^{*} \partial_{x} \Delta m_{x}-\frac{1}{\lambda^{2}} \Delta m_{z}=0 .
\end{aligned}
$$

Note that the source terms are absent $\delta$ n the right-hand side of Eqs. (A2) and (A3) unlike in Eqs. (22) and (23) for the in-plane longitudinal anisotropy case. The absence of the source terms implies that the DM torque vanishes up to the second order in spin-wave amplitude. This vanishing is actually consistent with the vector expressions of the DM torque presented in the main text; the fieldlike DM torque is proportional to $\mathbf{m} \times \mathbf{y}$ and the dampinglike DM torque is proportional to $\mathbf{m} \times(\mathbf{y} \times \mathbf{m})$, both of which vanish in the in-plane transverse anisotropy case since $\mathbf{m}$ is essentially aligned along the $\mathbf{y}$ direction.

\section{Perpendicular anisotropy}

In this case, $K_{x}=0, K_{y}=0$ and $K_{z}=-K_{\perp}$ and the perpendicular anisotropy field is $H_{\perp}=2 K_{\perp} / M_{s}$. We assume that the magnetization is mostly oriented along the easy axis $\eta \mathbf{z}, \operatorname{so} \theta \ll 1$ and $\sin \theta \approx \eta \theta, \cos \theta \approx \eta$. Here $\theta$ is of the second order in spin-wave amplitude. In this case, the deviations are defined as $\Delta m_{x}=\eta \theta \cos \phi$ and $\Delta m_{y}=\eta \theta \sin \phi$. The equations are quite lengthy, but if one assumes a circular precession of the spin wave, i.e., $s_{\theta}^{0}=s_{\phi}^{0}$, the following relations are fulfilled:

$$
\left\langle s_{\theta}^{2}\right\rangle=\left\langle s_{\phi}^{2}\right\rangle,\left\langle s_{\theta} \partial_{x} s_{\theta}\right\rangle=\left\langle s_{\phi} \partial_{x} s_{\phi}\right\rangle,\left\langle s_{\theta} \partial_{x} s_{\phi}\right\rangle=-\left\langle s_{\phi} \partial_{x} s_{\theta}\right\rangle,
$$

and then, it is possible to obtain the nonlinear equation describing the spatial profile of the deviations,

$$
\begin{aligned}
\partial_{x}^{2} \Delta m_{x}-\frac{1}{\lambda_{\perp}^{2}} \Delta m_{x} & =-\eta \tilde{D}^{*}\left\langle s_{\theta} \partial_{x} s_{\theta}\right\rangle, \\
\partial_{x}^{2} \Delta m_{y}-\frac{1}{\lambda_{\perp}^{2}} \Delta m_{y} & =-\tilde{D}^{*}\left\langle s_{\theta} \partial_{x} s_{\phi}\right\rangle,
\end{aligned}
$$

where $\lambda_{\perp}^{2}=J / H_{\perp}$. Note that Eqs. (A4) and (A5) are essentially identical to Eqs. (22) and (23) for the in-plane longitudinal anisotropy case. Combined also with the result for the in-plane transverse anisotropy case, one finds that for all three types of the considered anisotropies, the DM torque can be expressed as a sum of the fieldlike torque $(\propto \mathbf{m} \times \mathbf{y})$ and the dampinglike torque $[\propto \mathbf{m} \times(\mathbf{y} \times \mathbf{m})]$.
[1] M. I. D’yakonov and V. I. Perel, JETP Lett. 13, 467 (1971); J. E. Hirsch, Phys. Rev. Lett. 83, 1834 (1999).

[2] M. Z. Hasan and C. L. Kane, Rev. Mod. Phys. 82, 3045 (2010); X.-L. Qi and S.-C. Zhang, ibid. 83, 1057 (2011).

[3] U. K. Rößler, A. N. Bogdanov, and C. Pfleiderer, Nature (London) 442, 797 (2006).

[4] I. M. Miron, G. Gaudin, S. Auffret, B. Rodmacq, A. Schuhl, S. Pizzini, J. Vogel, and P. Gambardella, Nat. Mater. 9, 230 (2010); I. M. Miron, K. Garello, G. Gaudin, P.-J. Zermatten, M. V.
Costache, S. Auffret, S. Bandiera, B. Rodmacq, A. Schuhl, and P. Gambardella, Nature (London) 476, 189 (2011).

[5] L. Liu, T. Moriyama, D. C. Ralph, and R. A. Buhrman, Phys. Rev. Lett. 106, 036601 (2011); Science 336, 555 (2012).

[6] A. Chernyshov, M. Overby, X. Liu, J. K. Furdyna, Y. LyandaGeller, and L. P. Rokhinson, Nat. Phys. 5, 656 (2009); D. Fang, H. Kurebayashi, J. Wunderlich, K. Výborný, L. P. Zârbo, R. P. Campion, A. Casiraghi, B. L. Gallagher, T. Jungwirth, and A. J. Ferguson, Nat. Nanotechnol. 6, 413 (2011). 
[7] Yu. A. Bychkov and E. I. Rashba, J. Phys. C: Solid State Phys. 17, 6039 (1984).

[8] K. Obata and G. Tatara, Phys. Rev. B 77, 214429 (2008); A. Manchon and S. Zhang, ibid. 78, 212405 (2008); I. Garate and A. H. MacDonald, ibid. 80, 134403 (2009).

[9] X. Wang and A. Manchon, Phys. Rev. Lett. 108, 117201 (2012); K.-W. Kim, S. M. Seo, J. Ryu, K.-J. Lee, and H.-W. Lee, Phys. Rev. B 85, 180404 (2012); D. A. Pesin and A. H. MacDonald, ibid. 86, 014416 (2012); E. van der Bijl and R. A. Duine, ibid. 86, 094406 (2012).

[10] P. M. Haney, H. W. Lee, K. J. Lee, A. Manchon, and M. D. Stiles, Phys. Rev. B 87, 174411 (2013).

[11] I. E. Dzyaloshinskii, Sov. Phys. JETP 5, 1259 (1957); T. Moriya, Phys. Rev. 120, 91 (1960).

[12] M. Bode, M. Heide, K. von Bergmann, P. Ferriani, S. Heinze, G. Bihlmayer, A. Kubetzka, O. Pietzsch, S. Blügel, and R. Wiesendanger, Nature (London) 447, 190 (2007); P. Ferriani, K. von Bergmann, E. Y. Vedmedenko, S. Heinze, M. Bode, M. Heide, G. Bihlmayer, S. Blügel, and R. Wiesendanger, Phys. Rev. Lett. 101, 027201 (2008).

[13] A. N. Bogdanov and U. K. Rößler, Phys. Rev. Lett. 87, 037203 (2001).

[14] G. Chen, J. Zhu, A. Quesada, J. Li, A. T. N'Diaye, Y. Huo, T. P. Ma, Y. Chen, H. Y. Kwon, C. Won, Z. Q. Qiu, A. K. Schmid, and Y. Z. Wu, Phys. Rev. Lett. 110, 177204 (2013).

[15] A. Thiaville, S. Rohart, E. Jué, V. Cros, and A. Fert, Europhys. Lett. 100, 57002 (2012).

[16] K.-W. Kim, H.-W. Lee, K.-J. Lee, and M. D. Stiles, Phys. Rev. Lett. 111, 216601 (2013); A. Crepieux and C. Lacroix, J. Magn. Magn. Mater. 182, 341 (1998).

[17] S. Emori, U. Bauer, S.-M. Ahn, E. Martinez, and G. S. D. Beach, Nat. Mater. 12, 611 (2013).

[18] K.-S. Ryu, L. Thomas, S.-H. Yang, and S. Parkin, Nat. Nanotechnol. 8, 527 (2013).

[19] V. V. Kruglyak, S. O. Demokritov, and D. Grundler, J. Phys. D: Appl. Phys. 43, 264001 (2010); B. Lenk, H. Ulrichs, F. Garbs, and M. Münzenberg, Phys. Rep. 507, 107 (2011).

[20] K. Uchida, J. Xiao, H. Adachi, J. Ohe, S. Takahashi, J. Ieda, T. Ota, Y. Kajiwara, H. Umezawa, H. Kawai, G. E. W. Bauer, S. Maekawa, and E. Saitoh, Nat. Mater. 9, 894 (2010).

[21] Y. Kajiwara, K. Harii, S. Takahashi, J. Ohe, K. Uchida, M. Mizuguchi, H. Umezawa, H. Kawai, K. Ando, K. Takanashi, S. Maekawa, and E. Saitoh, Nature (London) 464, 262 (2010)

[22] J. Torrejon, G. Malinowski, M. Pelloux, R. Weil, A. Thiaville, J. Curiale, D. Lacour, F. Montaigne, and M. Hehn, Phys. Rev. Lett. 109, 106601 (2012); W. Jiang, P. Upadhyaya, Y. Fan,
J. Zhao, M. Wang, L.-T. Chang, M. Lang, K. L. Wong, M. Lewis, Y.-T. Lin, J. Tang, S. Cherepov, X. Zhou, Y. Tserkovnyak, R. N. Schwartz, and K. L. Wang, ibid. 110, 177202 (2013).

[23] A. Kovalev and Y. Tserkovnyak, Europhys. Lett. 97, 67002 (2012); P. Yan, X. S. Wang, and X. R. Wang, Phys. Rev. Lett. 107, 177207 (2011); D. Hinzke and U. Nowak, ibid. 107, 027205 (2011).

[24] L. Kong and J. Zang, Phys. Rev. Lett. 111, 067203 (2013).

[25] M. Hatami, G. E. W. Bauer, Q. Zhang, and P. J. Kelly, Phys. Rev. Lett. 99, 066603 (2007).

[26] G. E. W. Bauer, E. Saitoh, and B. J. van Wees, Nat. Mater. 11, 391 (2012).

[27] Y. Onose, T. Ideue, H. Katsura, Y. Shiomi, N. Nagaosa, and Y. Tokura, Science 329, 297 (2010); R. Matsumoto and S. Murakami, Phys. Rev. Lett. 106, 197202 (2011).

[28] Kh. Zakeri, Y. Zhang, J. Prokop, T.-H. Chuang, N. Sakr, W. X. Tang, and J. Kirschner, Phys. Rev. Lett. 104, 137203 (2010).

[29] J.-H. Moon, S.-M. Seo, K.-J. Lee, K.-W. Kim, J. Ryu, H.-W. Lee, R. D. McMichael, and M. D. Stiles, Phys. Rev. B 88, 184404 (2013).

[30] See, e.g., F. Schubert, Y. Mokrousov, P. Ferriani, and S. Heinze, Phys. Rev. B 83, 165442 (2011).

[31] D. V. Berkov and N. L. Gorn, J. Appl. Phys. 99, 08Q701 (2006).

[32] S.-M. Seo, K.-J. Lee, H. Yang, and T. Ono, Phys. Rev. Lett. 102, 147202 (2009).

[33] S. Zhang, P. M. Levy, and A. Fert, Phys. Rev. Lett. 88, 236601 (2002); S. Zhang and Z. Li, ibid. 93, 127204 (2004).

[34] X. Fan, H. Celik, J. Wu, C. Ni, K.-Jin Lee, V. O. Lorenz and J. Q. Xiao, Nat. Commun. 5, 3042 (2014).

[35] J. Xia, Y. Maeno, P. T. Beyersdorf, M. M. Fejer, and A. Kapitulnik, Phys. Rev. Lett. 97, 167002 (2006).

[36] S. S.-L. Zhang and S. Zhang, Phys. Rev. Lett. 109, 096603 (2012).

[37] M. Schreier, A. Kamra, M. Weiler, J. Xiao, G. E. W. Bauer, R. Gross, and S. T. B. Goennenwein, Phys. Rev. B 88, 094410 (2013) and references therein.

[38] W. F. Brown, Jr., IEEE Trans. Magn. 15, 1196 (1979).

[39] G. Grinstein and R. H. Koch, Phys. Rev. Lett. 90, 207201 (2003).

[40] S. Mühlbauer, B. Binz, F. Jonietz, C. Pfleiderer, A. Rosch, A. Neubauer, R. Georgii, and P. Böni, Science 323, 915 (2009); X. Z. Yu, Y. Onose, N. Kanazawa, J. H. Park, J. H. Han, Y. Matsui, N. Nagaosa, and Y. Tokura, Nature (London) 465, 901 (2010).

[41] S. Seki, X. Z. Yu, S. Ishiwata, and Y. Tokura, Science 336, 198 (2012).

[42] A. V. Chumak, A. A. Serga, and Burkard Hillebrands, Nat. Commun. 5, 4700 (2014). 